\title{
Anti-CD-20 Therapy in Refractory Adult Still's Disease
}

\author{
Reem Hamdy A. Mohammed \\ Rheumatology and Rehabilitation Department, Faculty of Medicine, Cairo University Hospitals, Taif, KSA. \\ Email: rmhamdy@yahoo.com
}

Received October $27^{\text {th }}, 2011$; revised November $22^{\text {nd }}, 2011$; accepted December $2^{\text {nd }}, 2011$

\begin{abstract}
Adult Still's disease is a relatively rare form of rheumatoid arthritis with systemic inflammatory features. The prevalence is around 1.5 cases per 100,000 - 1000,000. In the current case we display a 30-year-old male patient with refractory adult still's disease who suffered recurrent attacks of fever $39.5^{\circ} \mathrm{C}$, arthritis in proximal interphalangeal joints (PIPs), wrists, tempromandibular joints (TMJs), knees and ankles, stitching chest pain, dyspnea, erythematous rash over the trunk, sore throat, weight loss (15 Kilograms in 4 months). The patients' disease remained uncontrolled despite of synthetic disease modifying anti-rheumatic drugs and repeated intramuscular corticosteroid injections. Laboratory workup revealed erythrocyte sedimentation rate (ESR) of 95, C-reactive protein (CRP) of $100 \mathrm{mg} / \mathrm{L}$, hemoglobin 10.5 gm\%, leukocytosis 12,000/microlitre, mild elevation of liver function tests and dyslipidemia. Serology revealed negative rheumatoid factor, anti-nuclear antibody titre of 1:80, elevated serum ferritin 4000 micrograms/litre. The patient was started on rituximab ( $375 \mathrm{mg} / \mathrm{m}^{2}$ ), prednisolone $20 \mathrm{mg} /$ day and selective Cox-2 inhibitor. Follow up for over three months following the completion of his pulse therapy, revealed no relapse of fever or fatigue, with morning stiffness of 5 - 10 minutes, VAS of 3, DAS28 of 3.8, HAQDI of 0.62, ESR 23, CRP 4.9, Hb 12.5 gm\%, leucocytic count 9000/microlitre, and the dose of prednisolone was successfully reduced to a dose of $5 \mathrm{mg} /$ day orally. Conclusion: Anti-CD-20 therapy successfully controlled systemic and articular refractory disease with sustained efficacy over a follow up period of up to 24 weeks.
\end{abstract}

Keywords: Adult Still's Disease; Anti-TNF Therapy; Anti-CD-20 Therapy; Refractory Disease

\section{Introduction}

Adult onset Still's Disease (AoSD) is a relatively rare form of chronic inflammatory arthritis, that usually presents by high spiking fever, arthritis, myalgias, salmon pink skin rash, amongst other systemic inflammatory features. The prevalence is around 1.5 cases per 100,000 - 1000,000 population. The disease carries a characteristic bimodal age distribution 1st peak 15 - 25 years and a 2nd peak 35 - 45 years. The disease typically affects 16 35 years old and presents with arthralgia, elevated serum ferritin, "salmon-pink" rash, pyrexia and lymphadenopathy. Rheumatoid factor (RF) and anti-nuclear antibody (ANA) are classically negative. Patients who are experiencing a flare-up from Adult-onset Still's disease usually manifest with extreme fatigue, joint pain, swollen lymph nodes, and less commonly serositis with pleurisy and pericarditis. The etiology of AoSD remains unknown [1, 2]. At least seven sets of criteria have been proposed, the most sensitive criteria are the Yamaguchi criteria $[3,4]$. The Yamaguchi criteria are classified into four major criteria including: 1) Fever of at least $39 \mathrm{c}$ for at least one week, 2) Arthralgias or arthritis for at least two weeks, 3) Non-pruritic salmon colored rash usually on the trunk and the extremities at the time of fever, 4) Leukocytosis 10,000 /microlitre or greater, with granulocyte predominance and five minor criteria including: 1) Sore throat, 2) Lymphadenopathy, 3) Hepatomegaly or spelnomegally, 4) Abnormal liver function tests, 5) Negative tests for anti-nuclear antibody and rheumatoid factor. For establishing the diagnosis of AoSD the patient must satisfy 5 criteria with at least two major criteria [5].

\section{Case Report}

The current case displays a 30 year-old male patient presenting with recurrent attacks of intermittentfever $39.5{ }^{\circ} \mathrm{C}$, arthritis in proximal interphalangeal joints (PIPs), wrists, tempromandibular joints (TMJs), knees and ankles, stitching chest pain, dyspnea, erythematous rash over the trunk, sore throat, weight loss (15 Kilograms in 4 months).

\section{History of Present Illness}

The onset started 3-years ago by an acute attack of fever, pain in tempromandibular joints with difficulty in chewing, bilateral pain, tenderness and swelling over both wrists, hand joints, knees and ankles, with joint stiffness 
more than three hours in the morning, sore throat, lassitude, erythematous rash over the trunk, myalgia, parasternal unilateral stitching chest pain. The patient was admitted, and investigated. In view of these clinical findings and with exclusion of a possible infectious etiology or malignancy the patient was classified as a case of systemic onset rheumatoid arthritis (ASD). The patient was started on prednisolone $40 \mathrm{mg} / \mathrm{day}$, non-steroidal anti-inflammatory drug (Diclofenac $50 \mathrm{mg}$ t.i.d). Following control of pyrexia, methotrexate $20 \mathrm{mg}$ /week was added. During that time the patient reported some improvement but he couldn't perform activities of daily living in the same way as before with morning stiffness of 1 hour. The patient reported that the arthritis, fever and fatigue relapse despite being on treatment in a way that affected quality of life with increased absenteeism from work. Methotrexate was substituted for leflunomide at a dose of $20 \mathrm{mg} /$ day with some improvement, oral prednisolone dose was by then decreased to $10 \mathrm{mg}$ /day orally. After 4 months the patient came with the current presentation: fever $39.5^{\circ} \mathrm{C}$, bilateral symmetric arthritis of TMJs, wrists, PIPs, knees and ankles, sore throat, chest pain and abdominal pain with erythematous rash over the trunk. Physical examination: General examination: temperature: $39{ }^{\circ} \mathrm{C}$; blood pressure: $129 / 60$; respiratory rate: $22 /$ minute. The patient was conscious, oriented to time, place and persons with generalized pallor, cachexia, lassitude. Chest examination: tachypnea, harsh vesicular breathing with fine crepitation in the right middle and lower lung zones and pleural rub over the right parasternal area. Abdominal examination: salmon pink rash, mild hepatomegaly. Genitourinary examination was free apart from non-specific inguinal lymphadenopathy. Musculoskeletal examination revealed mild tenderness proximally in muscles of the upper and lower limbs. Bilateral symmetric arthritis with swelling and tenderness in TMJs, wrists, PIPs, knees, and ankles. Disease activity score in 28 joints (DAS-28) of 6.74, visual analogue scale for pain (VAS) of 7, multidimensional health assessment questionnaire disease index (MHAQ-DI) of 1.5. Laboratory investigations by that time revealed erythrocyte sedimentation rate (ESR) of 95, C-reactive protein (CRP) of $100 \mathrm{mg} / \mathrm{L}$, hemoglobin $10.5 \mathrm{gm} \%$, leukocytosis 12,000/ microlitre, mild elevation of liver function tests and dyslipidemia. Serology revealed rheumatoid factor of 15 $\mathrm{IU} / \mathrm{ml}$ (reference 0 - $30 \mathrm{IU} / \mathrm{ml}$ ), anti-nuclear antibody titre of 1:80, elevated serum ferritin 4000 micrograms/ Litre. Imaging studies showed areas of juxta-articular osteoporosis with small subchondral cystic lesions in PIPs and wrists, soft tissue swelling around the PIPs, wrists, knees and ankles. Chest $\times$ ray revealed pneumonitis with pleural effusion (sputum culture was negative for gram positive and negative organisms and acid fast bacilli), abdominal ultrasound revealed mild hepato-sple- nomegally.

\section{Management}

The dose of prednisolone was increased to $60 \mathrm{mg} / \mathrm{day}$ orally with a synchronous increase in the dose of oral non-steroidal anti-inflammatory drugs. Oral prednisolone was gradually tapered over a period of 4 weeks to 20 $\mathrm{mg} /$ day orally. Disease activity stayed uncontrolled with persistent systemic and articular disease, ESR 54, CRP 30 despite conventional DMARDs therapy, oral prednisolone and repeated intramuscular steroid injections, the patient was considered as a refractory disease.

The decision of starting the patient on biologic treatment was highly considered by that time. Anti-CD-20 therapy, rituximab was initiated $\left(375 \mathrm{mg} / \mathrm{m}^{2}\right)$ in addition to prednisolone $20 \mathrm{mg}$ /day and selective Cox-2 inhibitors. The treatment was initiated with an initial 2 doses of $1000 \mathrm{mg} /$ infusion with 2 weeks interval in between. Three months following the completion of his pulse therapy, the patient reported disappearance of fever, fatigue, morning stiffness was 5 - 10 minutes, the patient was able to pray and to perform his daily activities without difficulty, with VAS of 3, DAS28 3.8, HAQDI of 0.62, ESR 23, CRP 4.9, Hb 12.5 gm\%, leucocytic count 9000/ microlitre, the dose of prednisolone was successfully reduced to a dose of $5 \mathrm{mg} /$ day orally without reported relapse of pain or fever. Follow up of the patient after 6 month showed mild elevation of the ESR $30 \mathrm{~mm} / \mathrm{hr}$ and CRP $22.2 \mathrm{mg} / \mathrm{L}$, and mild pain with mild stiffness less than 10 minutes. The second pulse course was given after 6 months and the patient was satisfied with the outcome of therapy. Anti-CD-20 therapy in this case of refractory still's disease induced remission of clinical symptoms and signs of active disease with normalization of acute phase reactants (ESR, CRP) and improved quality of life with improved functional performance of the patient.

\section{Discussion}

Adult onset Still's Disease (AoSD) is a relatively rare form of chronic inflammatory arthritis in which the exact etiopathogenic and biologic contributors remains poorly understood with a recently recognized crucial role for cytokines like interleukin (IL)-1, IL-6 and IL-18, macrophage colony-stimulating factor, interferon-gamma and tumour necrosis factor (TNF)-alpha [1,6]. Yet, the wide range of disease manifestations and course suggests considerable heterogeneity of the disease entity and its pathogenetic background. The goal of treatment in patients with ASD is not solely focused on treating joint symptoms but also targets the systemic manifestations of the disease. With the new era of biologic therapy (Biologic disease modifying anti-rheumatic drugs), anti-tumor necrosis factor alpha therapy, anti-IL-1 therapy and 
recently anti-IL-6 are highly recommended in patients with chronic systemic activity who are considered refractory to traditional DMARDs [7-13]. Trials studying the use of anti-CD-20 (rituximab) in conventional DMARD non-responders with systemic onset rheumatoid arthritis (ASD) are lacking with only two case reports displaying efficacy of the drug in refractory cases who failed antiTNF therapy. In the current case we reported efficacy of anti-CD-20 therapy as first line biologic in methotrexate refractory ASD with satisfactory control of disease activity and a sustained response over a period of 24 weeks.

\section{Conclusion}

Despite that the exact role in disease modification with anti-CD-20 therapy hasn't been surveyed in these patients yet evidences have shown that following B cell depletion with rituximab treatment, a decrease was observed for IL-1b, IL-1RA, IL-2R, IL-4, IL-5, IL12, IL-15, IL-17, TNF- $\alpha$, GM-CSF for 24 weeks with partial recovery over a period of 24 - 48 weeks which suggests that B cell depletion might down regulate the release of $\mathrm{T}$ cell mediated pro-inflammatory cytokine release [9-13]. Considering that the exact pathogenesis of ASD remains poorly understood, therefore encouraging trials addressing the use of different lines of biologic disease modifying drugs in patients with AoSD might provide further insight into the pathogenesis of this complex disease.

\section{REFERENCES}

[1] P. Efthimiou, A. Kontzias, C. M. Ward and N. S. Ogden, “Adult-Onset Still's Disease: Can Recent Advances in Our Understanding of Its Pathogenesis Lead to Targeted Therapy?” Nature Reviews Rheumatology, Vol. 3, No. 6, 2007, pp. 328-335. doi:10.1038/ncprheum0510

[2] E. G. Bywaters, "Still's Disease in the Adult," Annals of Rheumatic Diseases, Vol. 30, No. 2, 1971, pp. 121-133. doi:10.1136/ard.30.2.121

[3] M. B. Owlia and G. Mehrpoor, “Adult-Onset Still's Disease: A Review," Indian Journal of Medical Sciences, Vol. 63, No. 5, 2009, pp. 207-221.

[4] F. Luthi, P. Zufferey, M. F. Hofer and A. K. So, “'Adolescent-Onset Still's Disease': Characteristics and Outcome in Comparison with Adult-Onset Still's Disease," Clinical and Experimental Rheumatology, Vol. 20, No. 3, 2002, pp. 427-430.
[5] M. Yamaguchi, A. Ohta, T. Tsunematsu, R. Kasukawa, Y. Mizushima, H. Kashiwagi, S. Kashiwazaki, K. Tanimoto, Y. Matsumoto and T. Ota, "Preliminary Criteria for Classification of Adult Still's Disease,” The Journal of Rheumatology, Vol. 19 , No. 3, 1992, pp. 424-430.

[6] A Kontzias and P. Efthimiou, “Adult-Onset Still's Disease: Pathogenesis, Clinical Manifestations and Therapeutic Advances,” Drugs, Vol. 68, No. 3, 2008, pp. 319337. doi:10.2165/00003495-200868030-00005

[7] P. Efthimiou and S. Georgy, "Pathogenesis and Management of Adult-Onset Still's Disease," Seminars in Arthritis and Rheumatism, Vol. 36, No. 3, 2006, pp. 144-152. doi:10.1016/j.semarthrit.2006.07.001

[8] I. Kötter, A. Wacker, S. Koch, J. Henes, C. Richter, A. Engel, I. Günaydin and L. Kanz, "Anakinra in patients with treatment-resistant adult-onset Still's disease: four case reports with serial cytokine measurements and a review of the literature," Seminars in Arthritis and Rheumatism, Vol. 37, No. 3, 2007, pp. 189-197. doi:10.1016/j.semarthrit.2007.04.002

[9] G. D. Kalliolias and S. N. Liossis, "The Future of the Il-1 Receptor Antagonist Anakinra: From Rheumatoid Arthritis to Adult-Onset Still's Disease and Systemic-Onset Juvenile Idiopathic Arthritis,” Expert Opinion on Investigational Drugs, Vol. 17, No. 3, 2008, pp. 349-359. doi:10.1517/13543784.17.3.349

[10] R. Thonhofer, M. Hiller, H. Just, M. Trummer, C. Siegel and C. Dejaco, "Treatment of refractory adult-onset still's disease with tocilizumab: report of two cases and review of the literature,” Rheumatology International, Vol. 31, No. 12, 2011, pp. 1653-1656. doi:10.1007/s00296-010-1631-y

[11] T. Naniwa, R. Ito, M. Watanabe, Y. Hayami, S. Maeda, K. Sasaki and S. Iwagaitsu, "Case Report: Successful Use of Short-Term Add-On Tocilizumab for Multirefractory Systemic Flare of Adult-Onset Still's Disease," Clinical Rheumatology, 14 September 2010, pp. 1-44. doi:10.1007/s10067-010-1562-8

[12] K. Ahmadi-Simab, P. Lamprecht, C. Jankowiak and W. L. Gross, "Successful Treatment of Refractory Adult Onset Still's Disease with Rituximab," Annals of the Rheumatic Diseases, Vol. 65, No. 8, 2006, pp. 1117-1118. doi:10.1136/ard.2005.047621

[13] E. Bartoloni, A. Alunno, F. Luccioli, G. Santoboni and R. Gerli, "Successful Treatment of Refractory Adult Onset Still's Disease with Anti-CD-20 Monoclonal Antibody," Clinical and Experimental Rheumatology, Vol. 27, No. 5, 2009, pp. 888-889. 